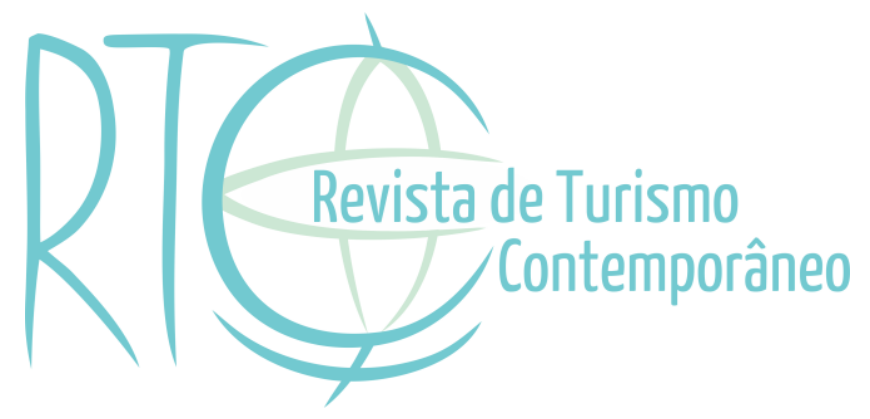

\title{
Escolha de destino para eventos: Uma análise dos atributos considerados na tomada de decisão por organizadores e promotores de eventos
}

Choice of destination for events: an analysis of attributes considered in the decision-making by organizers and event promoters

\section{Bruna Dorabiallo Oliveira}

Professora do Instituto Federal de Educação, Ciência e Tecnologia de Santa Catarina - IFSC, Florianópolis/SC, Brasil

E-mail: bdorabiallo@gmail.com

José Manoel Gonçalves Gândara

Professor do Departamento de Turismo da Universidade Federal do Paraná - UFPR, Curitiba/PR, Brasil

E-mail: jmggandara@yahoo.com.br

Bráulio Oliveira

Professor da Universidade Ceuma - UNICEUMA, São Luís/MA, Brasil

E-mail: braulioliveira@gmail.com 


\section{RESUMO}

O turismo de eventos provoca grande atratividade econômica para as localidades receptoras, uma vez que os eventos geram fluxo programado de visitantes e o turista tem um gasto médio superior aos demais tipos de turista. Compreender os atributos que a localidade deve apresentar para ser atrativa aos organizadores e promotores de eventos é uma maneira de planejar e desenvolver o turismo em uma região. Com o objetivo de analisar os conjuntos de atributos considerados na escolha de um destino para evento, o presente estudo apresenta uma pesquisa exploratória e através de pesquisa bibliográfica indica os estudos publicados nessa temática na perspectiva dos organizadores e promotores de eventos. Foram analisados 27 artigos que discutem os atributos inerentes à tomada de decisão de destinos para eventos e descritos os 21 diferentes conjuntos de atributos identificados. Verificou-se que se pode categorizar os atributos entre os relacionados ao evento ou ao destino, sendo que estrutura para eventos, hospedagem e custos são predominantes nos estudos, enquanto existem outros atributos são considerados por autores específicos.

Palavras-chave: Turismo. Eventos. Atributos. Promotores de Eventos. Organizadores de Eventos.

\section{ABSTRACT}

Event tourism generates great economic attractiveness to the localities that receive the events, because the events create a programed flow of visitors in addition of the tourist have an average spend greater than the other types of tourist. Understand the attributes that the destination must submit to be attractive to the organizers and promoters of events is a way to plan and develop the tourism in a region. With the objective of analyzing the sets of attributes used for choosing on the fate of an event, the present study presents an exploratory research and through bibliographic research indicates the studies published in this theme in the perspective of the organizers and promoters of events. They analyzed 27 articles that discuss the attributes of decision-making of destinations for events and described the 21 different sets of attributes identified. It was found that there are criteria as structure for events, accommodation and costs that are predominant in the studies, while other attributes are considered by specific authors.

Keywords: Tourism. Events. Attributes. Promoters of Events. Events Organizers. 


\section{INTRODUÇÃO}

O turismo é um fenômeno complexo, no qual há o envolvimento de diversos atores para o desenvolvimento da atividade (Barreto, 2003; Fratucci, 2014). A atuação desses diversos atores tem valor não só na fase de execução das ações, também é importante que cada ator assuma responsabilidades quanto ao planejamento, implementação e controle da atividade turística. (Gândara, Ruiz, Miki \& Biz, 2013). Dessa forma, percebe-se que a competitividade de cada ator está inter-relacionada com os atores envolvidos no processo (Buhalis, 2000).

A complexa relação dos atores, apresentada por Gândara et al. (2013), considera também aqueles que estão fora do destino e que podem induzir o fluxo turístico para determinada localidade. Um exemplo disso são os responsáveis por escolher um destino para evento e, por causa dessa escolha, tornam-se responsáveis por gerar fluxo de turistas para determinada localidade. Buhalis (2000) aponta ainda que a atuação integrada, com parcerias entre os atores do processo, é fundamental para a capacidade dos destinos em oferecer produtos adequados às necessidades de cada perfil. $\mathrm{O}$ envolvimento de um grande número de atores para a concretização das atividades do turismo fomenta a cadeia produtiva nas localidades e isso faz com que a atividade turística seja reconhecida como alternativa de desenvolvimento (Filipovic, 2012; Getz, 2008).

O turismo de eventos, sendo um segmento de turismo, também tem suas atividades relacionadas a uma complexa rede de atores públicos e privados. Essa tipologia é caracterizada como uma atividade que atrai turistas para uma localidade através do planejamento, desenvolvimento e comercialização de um acontecimento em um momento pré-agendado (Getz, 2008).

Enquanto o turismo busca compreender a viagem e o turista, o campo de estudos de eventos está relacionado à gestão de eventos e estudo de casos; relacionando as duas áreas tem-se que o turismo de eventos concentra seus estudos na relação entre o desenvolvimento dos destinos e o marketing para gestão dos eventos (Figura 1). A imagem dos destinos, a atratividade de cada localidade e a escolha pela sede dos eventos são exemplos de assuntos que se fundamentam tanto em conceitos de turismo quanto em teorias utilizadas na área de eventos. Mesmo o turismo de eventos tendo grande relevância turística os estudos nessa área ainda são pouco difundidos (Getz, 2008; Mair \& Thompson, 2009; Miki et al., 2013; Oppermann, 1996a). 


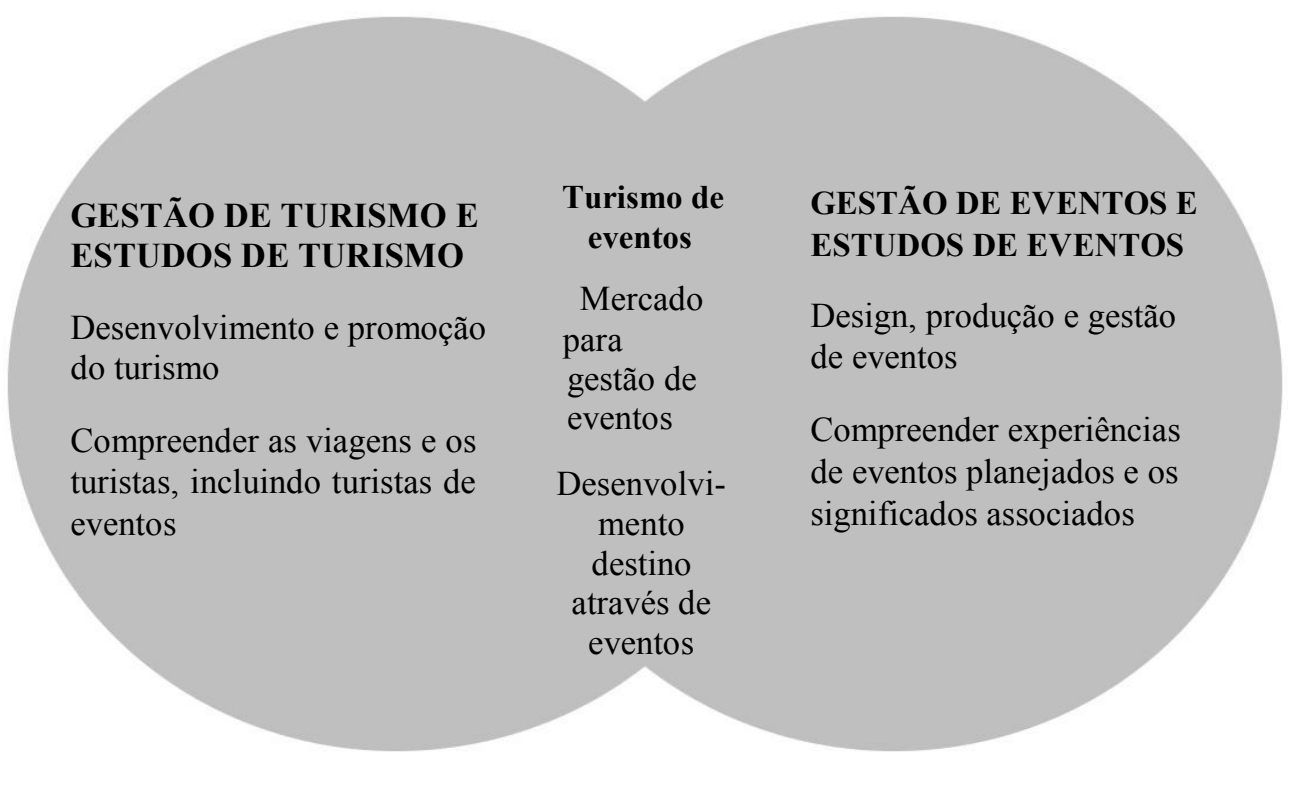

Figura 1 - Correlação entre estudos de turismo e de eventos Fonte: Getz, 2008, tradução nossa.

Eventos são importantes geradores de fluxo para a localidade, independente da época do ano em que ocorrem, uma vez que grande parte de sua atratividade está no fato de que os eventos são acontecimentos únicos e que só podem ser experenciados por quem nele estiver presente (Getz, 2008; Miki et al., 2013), além de ser uma forma de direcionar o fluxo de visitantes para períodos de menor procura turística e, com isso, reduzir a sazonalidade (Comas \& Moscardo, 2005; Crouch \& Louviere, 2004; Oppermann, 1996a, 1996b).

Com o impacto econômico positivo resultante do turismo de eventos, diversos destinos passam a dar importância a esse segmento, dado esse fato a concorrência entre os destinos vem aumentando (Baloglu \& Love, 2005; Crouch \& Louviere, 2007; Del Chiappa, 2012; DiPietro et al, 2008; Huo, 2014; Kim et al., 2011; Park et al., 2014). A grande concorrência entre os destinos se dá também pela característica de que eventos podem ocorrer em inúmeras localidades, desde que essas tenham características mínimas para atender ao evento (Chen, 2006; Crouch \& Louviere, 2004; Filipovic, 2012; Oppermann, 1996b).

Estabelecer critérios para que uma escolha seja feita em um ambiente em que há diversas opções é uma tarefa bastante complexa (Chen, 2006; Filipovic, 2012). A tomada de decisão já foi considerada uma ação exclusivamente racional (Filipovic, 2012), porém é necessário admitir que nesse processo haja além das influências cognitivas (racionais), a dimensão afetiva e a impressão global (Baloglu \& Love, 2005). 
Compreender os fatores que afetam o processo de escolha de um destino frente a seus concorrentes é essencial para que os atores do turismo de eventos possam trabalhar de maneira estratégica, atraindo mais eventos para o destino (Crouch \& Louviere, 2004, 2007; Elston \& Draper, 2012; Getz, 2004; Park et al., 2014). Para comunicar-se adequadamente com os tomadores de decisão sobre o destino de eventos é importante que as empresas compreendam o que esses organizadores e promotores desejam (Chen, 2006; Huo, 2014; Kang et al, 2005; Kim \& Weaver, 2010).

\begin{abstract}
Em um ambiente altamente competitivo, é imperativo para os destinos avaliar continuamente as suas forças e fraquezas. Eles devem identificar e corrigir as grandes deficiências na oferta de produtos e serviços, bem como encontrar seus ativos únicos diferenciadores que apelar para os participantes e organizadores de eventos (Park et al., 2014, p. 245, tradução nossa).
\end{abstract}

Ao desconhecer os atributos inerentes à escolha de um destino para eventos os gestores do destino desconhecem onde o como se devem investir seus recursos para melhorar a competitividade (Chen, 2006; Crouch \& Louviere, 2004).

Para compreender o processo de tomada de decisão dos organizadores e promotores sobre a escolha de um destino para eventos há diversos autores que desenvolveram estudos sobre os critérios da tomada de decisão (Quadro 1) e no presente estudo serão elencadas as variáveis presentes em cada pesquisa analisada, com o objetivo de analisar os conjuntos de atributos considerados na escolha de um destino para evento pelos organizadores e promotores de eventos.

Para atingir esse objetivo o estudo foi divido em etapas, conforme os tópicos que seguem. Através de um estudo exploratório, baseado em pesquisa bibliográfica realizada nos meses de janeiro e fevereiro de 2015, com revisão no mês de junho de 2015, o estudo foi conduzido. Primeiramente foram identificados alguns conceitos sobre turismo de eventos, eventos e tomada de decisão. Em um segundo momento foram elencados modelos de tomada decisão por organizadores e promotores de eventos para a escolha do destino. Após essa etapa foi realizada uma análise dos atributos identificados em cada modelo com base na metodologia de análise do conteúdo de Bardin (1977) e identificado que há duas categorias específicas de atributos: atributos relacionados ao evento e atributos relacionados ao destino. Por fim, as considerações finais sobre os sensos e dissensos existentes são apresentadas. 


\title{
2. METODOLOGIA
}

A importância do turismo de eventos ultrapassa o evento em si, mobilizando a cadeia de atores e gerando impactos para a comunidade em que esse acontecimento é realizado (Filipovic, 2012; Getz, 2008). A metodologia utilizada para o desenvolvimento desse estudo baseou-se em atingir o objetivo de analisar os conjuntos de atributos considerados na escolha de um destino para evento pelos organizadores e promotores de eventos, para tanto foi realizado um estudo exploratório, baseado em pesquisa bibliográfica e de caráter qualitativo. De acordo com Gil (2007, p. 44):

\begin{abstract}
A pesquisa bibliográfica é desenvolvida a partir de material já elaborado, constituído principalmente de livros e artigos científicos. Embora em quase todos os estudos seja exigido algum tipo de trabalho desta natureza, há pesquisas desenvolvidas exclusivamente a partir de fontes bibliográficas. Boa parte dos estudos exploratórios pode ser definida como pesquisas bibliográficas. A principal vantagem da pesquisa bibliográfica reside no fato de permitir ao investigador a cobertura de uma gama de fenômenos muito mais ampla do que aquela que poderia pesquisar diretamente. Esta vantagem se torna particularmente importante quando o problema de pesquisa requer dados muito dispersos pelo espaço.
\end{abstract}

O uso do caráter qualitativo do estudo caracteriza-se na interpretação de dados abertos com interpretação pessoal da pesquisadora (Creswell, 2007). A pesquisa bibliográfica foi realizada através de um levantamento e posterior análise de artigos científicos disponíveis nas seguintes bases de dados: Periódicos CAPES, Publicações de turismo, Redalyc e ScienceDirect. O período de coleta de dados nas bases citadas ocorreu entre os meses de janeiro e fevereiro de 2015, com revisão no mês de junho de 2015. Em toda pesquisa foi utilizado como parâmetro geral de busca a opção "artigos científicos" e considerado o recorte temporal dos últimos 10 anos.

As pesquisas foram realizadas com base em palavras-chave, seguindo em todas as bases utilizadas a combinação em inglês, português e espanhol dos termos: turismo, modelagem, decisores, tomada de decisão, organizador de eventos, promotor de eventos, eventos, turismo de eventos, MICE, convenções.

Após a identificação dos os artigos que continham temas correlatos aos tópicos pesquisados foram analisados se nas referências havia indicação de materiais adicionais com assunto correlato, como em um efeito "bola de neve".

Em um primeiro momento as pesquisas reuniram um total de 276 artigos, após análise das palavras-chave e resumo foram selecionados 72 artigos e excluídos aqueles que não estavam relacionados à escolha de destinos para eventos. Após a análise do conteúdo 
foram classificados 27 artigos correspondentes ao tema de estudo desse artigo, os quais estão discriminados no quadro 1.

Quadro 1 - Artigos analisados e descritos nesse estudo

\begin{tabular}{|c|c|c|c|}
\hline TÍTULO & AUTORES & ANO & PERIÓDICO \\
\hline $\begin{array}{l}\text { Meeting Planners' Perception on Convention } \\
\text { Destination Attributes: Empirical Evidence } \\
\text { from Six Major Asian Convention Cities }\end{array}$ & Yang Huo & 2014 & $\begin{array}{c}\text { The Journal of Business } \\
\text { Inquiry }\end{array}$ \\
\hline $\begin{array}{c}\text { The Great Halls of China? Meeting Planners' } \\
\text { Perceptions of Beijing as an International } \\
\text { Convention Destination }\end{array}$ & $\begin{array}{l}\text { Jinah Park, Bihu Wu, } \\
\text { Ye Shen, Alastair M. } \\
\text { Morrison \& Yeung } \\
\text { Kong } \\
\end{array}$ & 2014 & $\begin{array}{c}\text { Journal of Convention \& } \\
\text { Event Tourism }\end{array}$ \\
\hline $\begin{array}{c}\text { A Literature Review of Research on Site } \\
\text { Selection Criteria for Meeting and Exhibition } \\
\text { Planners }\end{array}$ & Yang Jingbo & 2013 & Tourism Tribune \\
\hline $\begin{array}{l}\text { A new prioritization method in the analytic } \\
\text { hierarchy process applied on a case study of } \\
\text { the convention site selection }\end{array}$ & $\begin{array}{l}\text { Milanka Gardašević } \\
\text { Filipović }\end{array}$ & 2012 & $\begin{array}{l}\text { JP Journal of Applied } \\
\text { Mathematics }\end{array}$ \\
\hline $\begin{array}{l}\text { A Review of Meeting Planner Site Selection } \\
\text { Criteria Research }\end{array}$ & $\begin{array}{l}\text { Kayla Elston e Jason } \\
\text { Draper }\end{array}$ & 2012 & $\begin{array}{c}\text { Journal of Convention \& } \\
\text { Event Tourism }\end{array}$ \\
\hline $\begin{array}{c}\text { How Do Meeting organizers choose } \\
\text { Convention sites } \\
\text { based on different types of Meetings? } \\
\text { An empirical analysis of the italian Meetings } \\
\text { industry } \\
\end{array}$ & Giacomo Del Chiappa & 2012 & Event Management \\
\hline $\begin{array}{l}\text { Competitive Positioning among International } \\
\text { Convention Cities in the East Asian Region }\end{array}$ & $\begin{array}{l}\text { Samuel Seongseop } \\
\text { Kim, Seunghyun Yoon } \\
\text { \&Youngmi Kim }\end{array}$ & 2011 & $\begin{array}{c}\text { Journal of Convention \& } \\
\text { Event Tourism }\end{array}$ \\
\hline $\begin{array}{c}\text { An Exploratory Study of Local Organizations' } \\
\text { Conference Choice Factors }\end{array}$ & $\begin{array}{c}\text { Beomcheol Peter Kim, } \\
\text { Dohee Kim, And } \\
\text { Pamela A. Weaver }\end{array}$ & 2010 & $\begin{array}{l}\text { Journal of Hospitality } \\
\text { Marketing \& } \\
\text { Management }\end{array}$ \\
\hline $\begin{array}{l}\text { An Exploratory Study of Differences among } \\
\text { Meeting and Exhibition Planners in their } \\
\text { Destination Selection Criteria }\end{array}$ & $\begin{array}{l}\text { Robin B. Di Pietro, } \\
\text { Deborah Breiter, Paul } \\
\text { Rompf e Marta } \\
\text { Godlewska } \\
\end{array}$ & 2008 & $\begin{array}{c}\text { Journal of Convention \& } \\
\text { Event Tourism }\end{array}$ \\
\hline $\begin{array}{c}\text { International Convention Site Selection: A } \\
\text { Further Analysis of Factor Importance Using } \\
\text { Best-worst Scaling }\end{array}$ & $\begin{array}{l}\text { Crouch G. e Louviere } \\
\text { J.J }\end{array}$ & 2007 & $\begin{array}{l}\text { Technical Report for } \\
\text { CRC for Sustainable } \\
\text { Tourism }\end{array}$ \\
\hline $\begin{array}{l}\text { Applying the Analytical Hierarchy Process } \\
\text { (AHP) Approach to Convention Site Selection }\end{array}$ & Ching-Fu Chen & 2006 & $\begin{array}{c}\text { Journal of Travel } \\
\text { Research }\end{array}$ \\
\hline $\begin{array}{l}\text { Understanding Associations and Their } \\
\text { Conference Decision-Making Processes }\end{array}$ & $\begin{array}{l}\text { Meghan Comas e } \\
\text { Gianna Moscardo }\end{array}$ & 2005 & $\begin{array}{c}\text { Journal of Convention \& } \\
\text { Event Tourism }\end{array}$ \\
\hline $\begin{array}{l}\text { Association meeting planners' perceptions and } \\
\text { intentions for five major US convention cities: } \\
\text { the structured and unstructured images }\end{array}$ & $\begin{array}{l}\text { Seyhmus Baloglu e } \\
\text { Curtis Love }\end{array}$ & 2005 & Tourism Management \\
\hline $\begin{array}{c}\text { The Competitiveness of International Meeting } \\
\text { Destinations in Asia: Meeting Planners' versus } \\
\text { Buying Centers' Perceptions }\end{array}$ & $\begin{array}{l}\text { Min-Hwi Kang, Seung- } \\
\text { Jin Suh e Deokhyun Jo }\end{array}$ & 2005 & $\begin{array}{c}\text { Journal of Convention \& } \\
\text { Event Tourism }\end{array}$ \\
\hline
\end{tabular}


Escolha de destino para eventos: Uma análise dos atributos considerados na tomada de decisão por organizadores e promotores de eventos

\begin{tabular}{|c|c|c|c|}
\hline $\begin{array}{l}\text { Determinants of destination choice in the } \\
\text { Australian domestic conventions sector }\end{array}$ & $\begin{array}{l}\text { Geoffrey Crouch e } \\
\text { Jordan Louviere }\end{array}$ & 2004 & $\begin{array}{l}\text { CRC for Sustainable } \\
\text { Tourism }\end{array}$ \\
\hline $\begin{array}{l}\text { The Analysis of Seoul as an International } \\
\text { Convention Destination }\end{array}$ & $\begin{array}{l}\text { Woo Gon Kim E } \\
\text { Hyeon-Cheol Kim }\end{array}$ & 2004 & $\begin{array}{l}\text { Journal of Convention \& } \\
\text { Exhibition Management }\end{array}$ \\
\hline $\begin{array}{c}\text { The Determinants of Convention Site } \\
\text { Selection: A Logistic Choice Model from } \\
\text { Experimental Data }\end{array}$ & $\begin{array}{l}\text { Geoffrey Crouch e } \\
\text { Jordan Louviere }\end{array}$ & 2004 & $\begin{array}{l}\text { Journal of Travel } \\
\text { Research }\end{array}$ \\
\hline $\begin{array}{l}\text { Association Meeting Planners' Perceived } \\
\text { Performance of Las Vegas. }\end{array}$ & $\begin{array}{l}\text { Seyhmus Baloglu e } \\
\text { Curtis Love }\end{array}$ & 2003 & $\begin{array}{l}\text { Journal of Convention \& } \\
\text { Exhibition Management }\end{array}$ \\
\hline $\begin{array}{c}\text { Selling Cities: Promoting New Images for } \\
\text { Meetings Tourism }\end{array}$ & $\begin{array}{c}\text { Andrew Bradley, Tim } \\
\text { Hall, Margaret } \\
\text { Harrison }\end{array}$ & 2002 & Cities \\
\hline $\begin{array}{l}\text { Determining the importance of US convention } \\
\text { destination attributes }\end{array}$ & $\begin{array}{l}\text { Harsha E. Chacko e } \\
\text { George G. Fenich }\end{array}$ & 2000 & $\begin{array}{l}\text { Journal of Vacation } \\
\text { Marketing }\end{array}$ \\
\hline $\begin{array}{c}\text { The comparative analysis of Hong Kong as an } \\
\text { international conference destination in } \\
\text { Southeast Asia }\end{array}$ & $\begin{array}{l}\text { Hailin Qu, Lan Li e } \\
\text { Gilder Kei Tat Chu }\end{array}$ & 2000 & Tourism Management \\
\hline $\begin{array}{l}\text { Classifying US association meeting planners } \\
\text { based on international destination selection } \\
\text { criteria: a case study of South Korea }\end{array}$ & $\begin{array}{l}\text { Jaekyoon Jun, Ken W. } \\
\text { McCleary }\end{array}$ & 1999 & Hospitality Management \\
\hline $\begin{array}{c}\text { Convention Site Selection Research: A } \\
\text { Review, Conceptual Model, and Propositional } \\
\text { Framework }\end{array}$ & $\begin{array}{l}\text { Geoffrey I. Crouch e J. } \\
\text { R. Brent Ritchie }\end{array}$ & 1997 & $\begin{array}{l}\text { Journal of Convention \& } \\
\text { Exhibition Management }\end{array}$ \\
\hline $\begin{array}{c}\text { Convention Cities - Images and Changing } \\
\text { Fortunes }\end{array}$ & Martin Oppermann & 1996 & $\begin{array}{c}\text { The Journal of Tourism } \\
\text { Studies } \\
\end{array}$ \\
\hline $\begin{array}{l}\text { Convention destination images: analysis of } \\
\text { association meeting planners' perceptions }\end{array}$ & Martin Oppermann & 1996 & Tourism Management \\
\hline $\begin{array}{l}\text { Quality service issues: a multivariate analysis } \\
\text { of association-meeting planner perceptions of } \\
\text { Caribbean destinations }\end{array}$ & $\begin{array}{l}\text { Mark A. Bonn, Jane } \\
\text { Boyd Ohlin e Richard } \\
\text { R. Brand }\end{array}$ & 1994 & $\begin{array}{l}\text { Journal of Hospitality \& } \\
\text { Tourism Research }\end{array}$ \\
\hline $\begin{array}{l}\text { What meeting planners want: the conjoint- } \\
\text { analysis approach }\end{array}$ & $\begin{array}{c}\text { Leo M. Renaghan e } \\
\text { Michael Z. Kay }\end{array}$ & 1987 & $\begin{array}{l}\text { The Cornell H. R. A. } \\
\text { Quarterly }\end{array}$ \\
\hline
\end{tabular}

\section{Fonte: Elaborado pelos autores, 2016.}

Entre os 27 estudos analisados nesse trabalho, foram encontrados 21 diferentes conjuntos de atributos, apresentados no quadro 3, que abordavam especificamente o processo de tomada de decisão dos organizadores e promotores de eventos na escolha do destino e os atributos considerados nessa decisão. A disparidade entre o número de artigos e o número de conjuntos de atributos se dá por 6 dos estudos utilizarem integralmente e citarem conjuntos de atributos apresentados em outros trabalhos.

As interpretações e inferências realizadas nesse estudo buscaram seguir a metodologia de análise do conteúdo de Bardin (1977). O corpus dessa análise foi formado 
pelos conjuntos de atributos identificados (Quadro 3) dos artigos analisados nesse estudo (Quadro 1). Após extensiva leitura do corpus da pesquisa foram identificadas algumas unidades de registro (Bardin, 1977) de acordo com a frequência de exposição ou importância do tema de acordo com a revisão de literatura. Essas unidades de registro foram categorizadas de acordo com as correlações identificadas entre atributos relacionados ao evento ou ao destino.

Quadro 2-Categorias de análise

\begin{tabular}{|c|c|}
\hline CATEGORIAS & UNIDADES DE REGISTRO \\
\hline Atributos do evento & $\begin{array}{c}\text { Estrutura disponível para o evento, qualidade das instalações do evento, apoio } \\
\text { local para a realização do evento (entidade receptora, governo local, CVB), custos } \\
\text { para a organização do evento, riscos para a realização do evento, ineditismo na } \\
\text { escolha do destino, sustentabilidade do evento. }\end{array}$ \\
\hline Atributos do destino & $\begin{array}{c}\text { Hospedagem, alimentação, custos dos produtos e serviços no destino, qualidade } \\
\text { dos produtos e serviços no destino, experiência na recepção/promoção de eventos, } \\
\text { imagem e/ou reputação do destino, clima, acessibilidade, segurança, opços de } \\
\text { entretenimento, questões legais para o acesso ao destino, hospitalidade, } \\
\text { sustentabilidade no destino }\end{array}$ \\
\hline
\end{tabular}

Fonte: Elaborado pelos autores, 2017.

Por fim, as categorias identificadas e conjuntos de atributos foram discutidas com base na revisão de literatura realizada para esse estudo. As inferências sobre essas categorias, bem como as considerações, sensos e dissensos visualizados na análise comparativa dos conjuntos elencados são expostos no tópico de análise desse artigo.

\section{OS ATRIBUTOS RELACIONADOS À ESCOLHA DO DESTINO DE EVENTOS NA PERSPECTIVA DOS ORGANIZADORES E PROMOTORES}

Ao compreender os processos envolvidos na tomada de decisão os destinos aumentam as chances de sucesso na atratividade de eventos, fornecendo informações personalizadas e buscando atender necessidades pontuais de acordo com as características do destino (Chacko \& Fenich, 2000; Elston \& Draper, 2012; Huo, 2014; McCleary, 1995). Dessa forma, tanto os responsáveis por escolher o destino como aqueles que o promovem e vendem devem compreender os atributos e o processo de escolha como forma de aperfeiçoar sua atuação (Crouch \& Ritchie, 1998; DiPietro et al., 2008; Oppermann, 1996b). Kim et al. (2010) ampliam essa discussão ao apontar que quando compradores e vendedores conhecem 
os atributos que influenciam a decisão haverá uma comunicação com mais clareza e facilidade entre eles.

Cada destino é único, formado por um conjunto de influências e características em inúmeros aspectos que o diferencia de todas as outras localidades. Ao pesquisar um destino para evento os tomadores de decisão procuram congregar as necessidades do evento com as características de uma localidade (Chen, 2006; Crouch \& Louviere, 2004; Filipovic, 2012). As características de um destino para eventos que podem influenciar a escolha da localidade são nomeadas de diferentes formas nas pesquisas da área, não havendo consenso quanto a isso. Para nomear essas características de um destino são utilizadas diferentes nomenclaturas, como: atributos, fatores, critérios, variáveis. Identificou-se que atributo é a nomenclatura mais aplicada nos estudos dessa área (Baloglu \& Love, 2003, 2005; Chen, 2006; Comas \& Moscardo, 2005; Crouch \& Louviere, 2004, 2007; Getz, 2004; Huo, 2014; Kim E Kim, 2004; Kang et al., 2005; Kim et al., 2010; Kim et al., 2011; Oppermann, 1996a, 1996b; Huo, 2014), portanto o presente estudo adotou-se atributo como a termo que referencia as características de determinado destino para eventos

Cada destino deve buscar identificar seus atributos, para dessa forma poder comunicar adequadamente quais são seus diferencias (Chako \& Fenich, 2000; Comas \& Moscardo, 2005; Elston \& Draper, 2012; Kim et al., 2010).

Compreendendo a importância de se conhecer os atributos que causam influência para os diversos atores envolvidos no turismo de eventos, a seguir serão apresentados estudos que discutiram os atributos relevantes para a escolha de um destino de eventos. O quadro 3 resume os 21 conjuntos de atributos apresentados por cada um dos estudos hora analisados e que posteriormente foram analisados.

Quadro 3 - Atributos considerados por organizadores e promotores de eventos na escolha do destino

\begin{tabular}{|c|c|}
\hline ESTUDOS & ATRIBUTOS \\
\hline $\begin{array}{c}\text { Fortin, Ritchie } \\
\text { e Arsenault } \\
(1976)\end{array}$ & $\begin{array}{c}\text { Experiência anterior, Acessibilidade, Apoio local, Oportunidades extra para o evento, } \\
\text { Instalações de hospedagem, Instalações para o evento, Informação, Ambiente local, } \\
\text { Outros critérios }\end{array}$ \\
\hline $\begin{array}{c}\text { ASAE (1992) } \\
\text { Bonn et al. } \\
(1994)\end{array}$ & $\begin{array}{c}\text { Qualidade de serviço, instalações das salas de reuniões, acessibilidade, instalações dos } \\
\text { quartos, imagem local, jantar / entretenimento, transporte aéreo, instalações para } \\
\text { exposição, acessibilidade rodovias, localização geográfica, instalações recreativas, clima. }\end{array}$ \\
\hline Instalações e serviços: percepção de valor, percepção de serviço, orientação de valor, \\
conveniência. \\
Lazer: atividades em água, atividades em terra, atividades educacionais, relações com o
\end{tabular}




\begin{tabular}{|c|c|}
\hline & $\begin{array}{l}\text { meio ambiente. } \\
\text { Entretenimento: opções de eventos paralelos e opções de entretenimento local. }\end{array}$ \\
\hline $\begin{array}{c}\text { Edelstein e } \\
\text { Benini (1994) }\end{array}$ & $\begin{array}{c}\text { Disponibilidade de instalações, acesso ao local, custos de transporte, distância de } \\
\text { assistentes para o evento, clima, instalações de recreação, atrações turísticas, mandato } \\
\text { pelo estatuto, imagem local. }\end{array}$ \\
\hline $\begin{array}{l}\text { Oppermann } \\
\text { (1996) }\end{array}$ & $\begin{array}{l}\text { Instalações e espaços para eventos, qualidade dos serviços hoteleiros, disponibilidade de } \\
\text { UHs, limpeza e atratividade local, segurança, facilidade de transporte aéreo, custos de } \\
\text { alimentação e hospedagem, acessibilidade, imagem da cidade, custos com transporte, } \\
\text { instalações de restaurantes, instalações para exposições, cenário e oportunidades de } \\
\text { turismo, clima e vida noturna. }\end{array}$ \\
\hline $\begin{array}{c}\text { Crouch e } \\
\text { Ritchie (1998) }\end{array}$ & $\begin{array}{l}\text { Acessibilidade: custo, tempo, frequência de acessibilidade, conveniência e barreiras. } \\
\text { Apoio local: seção local, CVB/Centro de convenção e subsídios. } \\
\text { Oportunidades extra evento: entretenimento, compras, turismo, recreação, oportunidades } \\
\text { profissionais. } \\
\text { Instalações de hospedagem: capacidade, custo, serviço, segurança, disponibilidade. } \\
\text { Instalações para o evento: capacidade, layout, custo, ambiente, serviço, disponibilidade, } \\
\text { segurança. } \\
\qquad \underline{\text { Informação: experiência, reputação, marketing. }} \\
\text { Ambiente local: Clima, ambiente, infraestrutura, hospitalidade. } \\
\text { Outros critérios: riscos, rentabilidade, promoção da Associação, novidade. }\end{array}$ \\
\hline Qu et al. (2000) & $\begin{array}{l}\text { Hospedagem: disponibilidade e modernidade das instalações hoteleiras, os serviços do } \\
\text { hotel, preço/taxas do hotel. } \\
\text { Estrutura para eventos: disponibilidade e atualização de equipamento audiovisual, } \\
\text { serviços prestados pelo centro de convenções, disponibilidade de alimentos e serviços de } \\
\text { banquetes, disponibilidade de grandes centros de convenções, taxa de aluguel de local, } \\
\text { metragem quadrada disponível para a exposição, disponibilidade e atualização de vídeo- } \\
\text { link para teleconferência, disponibilidade de instalações de interpretação simultânea. } \\
\text { Outros: acessibilidade, segurança e limpeza, infraestrutura, imagem (política e } \\
\text { economia), organizadores de eventos profissionais, clima e meio ambiente, assistência de } \\
\text { viagem, custo de transporte, atrações e entretenimento. }\end{array}$ \\
\hline $\begin{array}{l}\text { Bradley et al. } \\
\qquad(2002)\end{array}$ & $\begin{array}{c}\text { Acessibilidade, qualidade e versatilidade dos espaços para eventos, serviço de } \\
\text { alimentação e banquetes, opções e qualidade dos serviços locais, localização e serviços } \\
\text { associados (lojas, hotéis, restaurantes), tecnologia e serviços tecnológicos, imagem da } \\
\text { cidade, experiências anteriores dos clientes. }\end{array}$ \\
\hline $\begin{array}{c}\text { Baloglu e Love } \\
(2003,2005)\end{array}$ & $\begin{array}{l}\text { Qualidade de restaurantes locais, variedade de restaurantes locais, variedade de } \\
\text { estabelecimentos comerciais, facilidade de transporte local, acessibilidade do destino, } \\
\text { restaurantes locais a preços acessíveis, capacidade de espaço para eventos, qualidade de } \\
\text { instalações para eventos (encontros/reuniões), qualidade de espaço para exposições, } \\
\text { número de UHs, espaço para exposições a preços acessíveis, tarifa de hospedagem a } \\
\text { preços acessíveis, legislação local, clima, número de hotéis a curta distância do centro de } \\
\text { convenções, atratividade para acompanhantes, variedade de atrações locais, reputação da } \\
\text { cidade, proteção e segurança de destino, serviços do CVB, patrocínio da CVB. }\end{array}$ \\
\hline Crouch e & Custo do local para evento, qualidade dos alimentos, salas de reunião/ plenária, \\
\hline
\end{tabular}




\begin{tabular}{|c|c|}
\hline $\begin{array}{l}\text { Louviere } \\
(2004)\end{array}$ & $\begin{array}{c}\text { hospedagem dentro/fora do local de evento, proximidade do local aos participantes, } \\
\text { espaço para exposição, Salas/espaços para pausas, taxas de hospedagem, ambiente físico, } \\
\text { oportunidades de entretenimento, passagem aérea sem restrições, configuração } \\
\text { sociocultural, equipamentos de áudio/vídeo, variedade de acomodações, salão de festas, } \\
\text { disponibilidade de táxi, clima esperado, melhores tarifas de passagem aérea, assistência } \\
\text { do Convention Bureau, assistência da seção local }\end{array}$ \\
\hline Kim e Kim & $\begin{array}{c}\text { Facilidade de acesso, sistema de transporte terrestre, facilidade de acesso por transporte } \\
\text { aéreo, preço razoável de serviços de alimentos e bebidas, vida noturna, simplicidade de } \\
\text { desembaraço aduaneiro, custo da viagem, apoio do governo, compras, atrações turísticas } \\
\text { e culturais, disponibilidade hoteleira, tarifa hoteleira, qualidade da comida, bebida e } \\
\text { serviço, disponibilidade de equipamentos de alta tecnologia para o evento, instalações } \\
\text { para o evento, eficiência da equipe gerencial em centros de evento, assistência no local } \\
\text { com organização e planejamento do evento, proteção e segurança, clima, imagem da } \\
\text { cidade, atratividade da área geográfica, gentileza e conhecimento da comunidade local } \\
\text { em língua estrangeira. }\end{array}$ \\
\hline $\begin{array}{l}\text { Kang et al. } \\
\text { (2005) }\end{array}$ & $\begin{array}{l}\begin{array}{l}\text { Acessibilidade: Disponibilidade de companhias aéreas e de transporte no local, Tempo e } \\
\text { esforço para chegar ao destino. }\end{array} \\
\begin{array}{c}\text { Atrações: compras, vida noturna, entretenimento, singularidade cultural e histórica, } \\
\text { recursos de turismo. }\end{array} \\
\text { Custos: custos de hospedagem, transporte, passeios e } \\
\text { restaurantes. }\end{array}$ \\
\hline Chen (2006) & $\begin{array}{l}\text { Instalações/ espaços para o evento e hospedagem: espaço, variedade de locais para o } \\
\text { evento e hospedagem, adequação das instalações, qualidade dos alimentos e bebidas. } \\
\begin{array}{r}\text { Custos: despesas de transporte, despesas de hospedagem, custo com alimentação e } \\
\text { bebidas, preços gerais dos produtos e serviços. }\end{array} \\
\frac{\text { Ambiente local: imagem da cidade, adequação e qualidade da infraestrutura local, }}{\text { acessibilidade. }} \\
\begin{array}{r}\text { Estrutura de apoio: apoio governamental, qualidade dos profissionais de eventos e } \\
\text { eficiência do setor de eventos. }\end{array} \\
\frac{\text { Oportunidades adicionais para o evento: clima, oportunidades de entretenimento, }}{\text { passeios e atrações culturais. }}\end{array}$ \\
\hline $\begin{array}{l}\text { Comas e } \\
\text { Moscardo } \\
(2005)\end{array}$ & $\begin{array}{c}\text { Acessibilidade: custo, tempo, frequência de acessibilidade, conveniência e barreiras. } \\
\text { Oportunidades extra evento: entretenimento, compras, turismo, recreação, oportunidades } \\
\text { profissionais. }\end{array}$ \\
\hline
\end{tabular}




\begin{tabular}{|c|c|}
\hline & 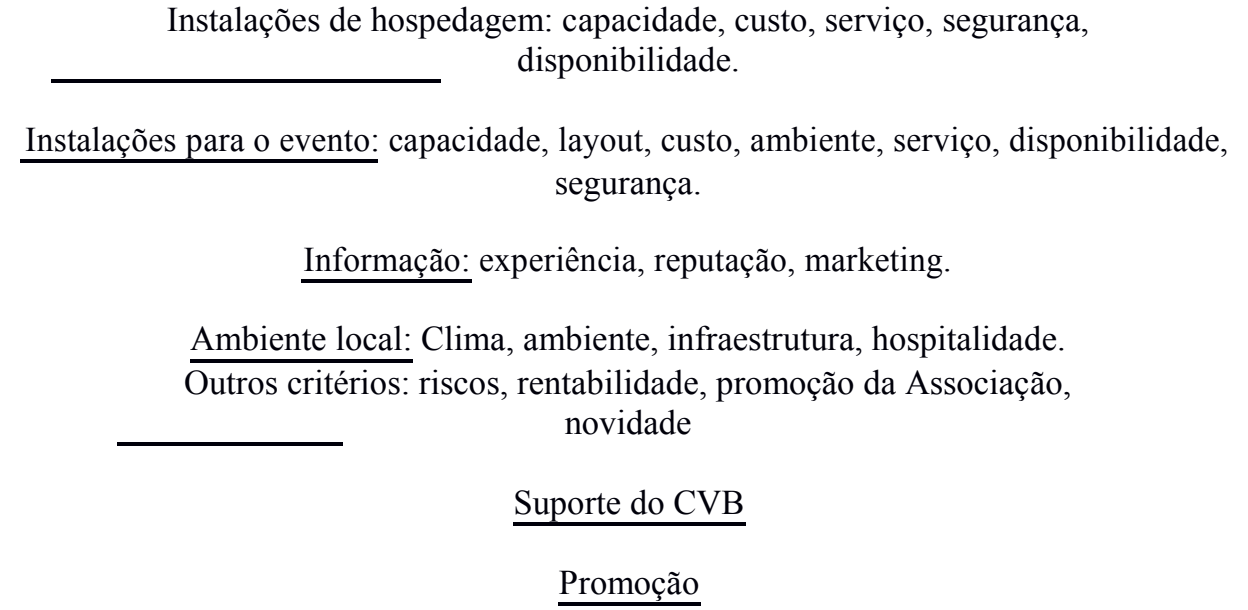 \\
\hline $\begin{array}{l}\text { Crouch e } \\
\text { Louviere } \\
(2007)\end{array}$ & $\begin{array}{l}\text { Frequência e de conveniência de horários e conexões de voo, qualidade da infraestrutura } \\
\text { geral, taxa de hospedagem para os participantes da conferência por alojamento, custo } \\
\text { local em relação à média internacional, espaços para os momentos de pausa/intervalo, } \\
\text { possibilidade de que possa haver subsídio para cobrir os custos, variedade e } \\
\text { disponibilidade de sistemas áudio/vídeo, capacidade da sala de eventos/ plenária, } \\
\text { oportunidades para networking profissional, ambiente e layout das instalações, barreiras } \\
\text { de viagem e formalidades (alfândega), nível de assistência esperado da seção local, } \\
\text { preocupações com a proteção e segurança dos participantes, porcentagem dos } \\
\text { participantes que podem ser acomodados no local do evento, nível de assistência } \\
\text { esperada do Convention \& Visitors local, hospitalidade e simpatia local, variedade de } \\
\text { hospedagem padrão três estrelas dentro de } 15 \text { minutos do local do evento, tempo desde a } \\
\text { última convenção no destino, economia média com passagens aéreas internacionais de } \\
\text { longa distância, economia média com passagens aéreas internacionais de curta distância, } \\
\text { percepção de confiabilidade / serviço ao cliente, risco potencial de interrupção do evento, } \\
\text { porcentagem de participantes que têm de viajar dentro do país, espaço de exposição, } \\
\text { porcentagem de participantes que têm de viajar uma longa distância, variedade de } \\
\text { hospedagem padrão cinco estrelas dentro de } 15 \text { minutos do local do evento, porcentagem } \\
\text { de participantes que vivem no local, ambiente físico exclusivo, ambiente cultural e social } \\
\text { únicos, oportunidades para roteiros turísticos / passeios, salão de festas/salas de jantar, } \\
\text { como foi a experiência anterior de realização do evento no destino, como é favorável a } \\
\text { reputação boca-a-boca do destino, variedade de hospedagem padrão quatro } \\
\text { estrelas } \\
\text { dentro de } 15 \text { minutos do local do evento, economia média com passagens aéreas } \\
\text { nacionais, qualidade percebida da comida, tempo típico e clima na época da convenção, } \\
\text { localização relativa dos locais de hospedagem para o aeroporto, oportunidades para } \\
\text { atividades recreativas, oportunidades para entretenimento, oportunidades para fazer } \\
\text { compras. }\end{array}$ \\
\hline $\begin{array}{l}\text { Di Pietro et al. } \\
\quad(2008)\end{array}$ & $\begin{array}{l}\text { Acessibilidade por via aérea, acessibilidade por estrada, opções de restaurante, variedade } \\
\text { de atividades noturnas, quartos de primeira classe em hotéis, marcas dos hotéis, espaço } \\
\text { para eventos, imagem de destino, reputação de hospedagem eventos bem-sucedidos, } \\
\text { proteção e segurança, serviços de apoio para eventos, custo e poder de compra no destino }\end{array}$ \\
\hline $\begin{array}{l}\text { Kim et al. } \\
(2010)\end{array}$ & $\begin{array}{c}\text { Despesas com hospedagem, preço dos alimentos, fácil acesso, instalações para } \\
\text { eventos/salas de reunião, Instalação dos restaurantes, alimentos e bebidas, Relações e } \\
\text { parceria, Referência, instalações de recreação, segurança, qualidade do serviço, } \\
\text { disponibilidade, Satisfação prévia com as instalações, satisfação prévia com os serviços, } \\
\text { atratividade local, Reputação. }\end{array}$ \\
\hline Kim et al. & $\begin{array}{l}\text { Instalações adequadas de hospedagem, segurança, opções de atrações turísticas, imagem } \\
\text { da cidade, preços competitivos, clima, receptividade da comunidade local }\end{array}$ \\
\hline $\begin{array}{l}\text { Del Chiappa } \\
\quad(2012)\end{array}$ & $\begin{array}{l}\text { Salas de reuniões e disponibilidade de equipamento audiovisual moderno, instalações de } \\
\text { exposições, disponibilidade de centro de convenções de grande porte, variedade de } \\
\text { hospedagem, tarifa de hospedagem, instalações de hospedagem, número de hotéis a uma }\end{array}$ \\
\hline
\end{tabular}




\begin{tabular}{|c|c|}
\hline & $\begin{array}{c}\text { curta distância do centro de convenções, hospedagem dentro/fora do local de evento, } \\
\text { localização dos locais de hospedagem em relação ao aeroporto, instalações dos } \\
\text { restaurantes (custo, qualidade e variedade), custos de transporte local e tempo de viagem, } \\
\text { acessibilidade do destino, imagem do destino, percepção do destino, novidade do destino, } \\
\text { proteção e segurança, clima esperado, configurações físicas e socioculturais, } \\
\text { entretenimento e oportunidade extra evento (lojas, teatros, museus), assistência do } \\
\text { Convention \& Visitors Bureau, qualidade e eficiência do pessoal da área de eventos, } \\
\text { preços acessíveis no geral. }\end{array}$ \\
\hline Huo (2014) & $\begin{array}{l}\begin{array}{c}\text { Instalações para eventos: Tamanho do espaço e sua disponibilidade, Condição e } \\
\text { qualidade, adequação dos estabelecimentos, variedade de locais, reputação (imagem), } \\
\text { tarifas de aluguel }\end{array} \\
\begin{array}{r}\text { Ambiente do destino: Acessibilidade da cidade, imagem do País (cidade), adequação, } \\
\text { infraestrutura. }\end{array} \\
\text { Instalações de hospedagem: espaço e disponibilidade dos locais para eventos, condição e } \\
\text { qualidade, tarifas de hotéis, adequação das instalações, prestatividade dos atendentes, } \\
\text { reputação. } \\
\text { Custos: hotel, tarifa aérea, aluguel do espaço para evento, alimentação, aluguel de } \\
\text { equipamentos, transporte local. } \\
\text { Apoio local: suporte do governo (CVB), qualidade de pessoal do setor, eficiência do } \\
\text { pessoal do setor, prestatividade do pessoal de serviço. } \\
\text { Outros fatores: segurança de participantes, habilidade de comunicação dos trabalhadores, } \\
\text { simpatia da comunidade local, interação com CMP local (PCO). }\end{array}$ \\
\hline $\begin{array}{l}\text { Park et al. } \\
\text { (2014) }\end{array}$ & $\begin{array}{l}\text { Características do destino: acessibilidade geográfica, adequação e padrão } \\
\text { de } \\
\text { infraestrutura local, Transporte terrestre local, Imagem da Cidade, atrações turísticas, } \\
\text { Clima, habilidade em Língua estrangeira da população local, opções de acomodações, } \\
\text { atratividade como um destino de eventos internacional, criação de publicidade da mídia. } \\
\text { Instalações para convenções e oportunidades de parceria: Ampla opção de escolha de } \\
\text { locais para o evento, Qualidade das instalações para o evento, oportunidades de } \\
\text { cooperação em Pesquisa e desenvolvimento. } \\
\text { Experiência e serviços: Qualidade de Serviços de eventos, Comida e bebida de qualidade, } \\
\text { Qualidade da assistência no planejamento do evento, experiência em sediar eventos } \\
\text { internacionais } \\
\text {. } \\
\frac{\text { Níveis de preços: custo total da realização de um evento, Custo das tarifas aéreas. }}{\text { Governo e políticas: Conveniência do processo de candidatura, apoio do governo, }} \\
\text { estabilidade política e social, formalidades de entrada (alfândega). }\end{array}$ \\
\hline
\end{tabular}

Fonte: Organizado pelos autores, 2016 com base em Asae (1992); Baloglu e Love (2003, 2005); Bradley et al. (2002); Bonn et al. (1994); Chacko e Fenich, (2000); Chen (2006); Clarck e McCleary (1995); Comas e Moscardo (2005); Crouch e Louviere, (2004, 2007); Crouch e Ritchie (1998); Del Chiappa (2012); Dipietro et al. (2008); Edelstein e Binini (1994); Elston e Draper (2012); Filipovic (2012); Fortin, Ritchie e Arsenault (1976); Huo (2014); Kang et al. (2005); Kim e Kim (2004); Kim et al. (2010); Kim et al. (2011);

Oppermann (1996a, 1996b); Park et al. (2014), Qu et al. (2000); Renaghan e Kay (1987); Yang (2013). 
Visualiza-se que diversos autores desenvolveram trabalhos em que foram elencados conjuntos de atributos e sua importância relativa no processo de escolha de um destino de eventos foi discutida. No tópico de análise desse trabalho serão apresentadas as relações visualizadas entre os conjuntos de atributos acima descritos.

\section{ANÁLISE DOS CONJUNTOS DE ATRIBUTOS CONSIDERADOS NA ESCOLHA DO DESTINO PELOS ORGANIZADORES E PROMOTORES DE EVENTOS}

Ao realizar uma análise comparativa dos atributos apresentados nos estudos já descritos é possível indicar algumas complementaridades, inovações e também atributos que são indicados de maneira pontual. Os atributos relacionados à estrutura para o evento e hospedagem são unânimes entre os modelos, sendo assim percebe-se que há igual importância tanto na categoria de atributos do destino quanto na do evento. Questões relacionadas especificamente à qualidade das instalações para evento, hotéis e restaurantes surge pela primeira vez no modelo da ASAE (1992) e posteriormente permeiam a maior parte dos estudos, estando presente em Oppermann (1996a, 1996b), Bradley et al. (2002), Baloglu e Love (2003, 2005), Crouch e Louviere (2004, 2007), Kim e Kim (2004), Kang et al. (2005), Chen (2006), Del Chiappa (2012), Huo (2014) e Park et al. (2014).

A experiência anterior de um destino em receber eventos de uma determinada empresa ou associação e a experiência dos organizadores e promotores com o destino foi um atributo citado apenas por Fortin, Ritchie e Arsenault (1976), Crouch e Ritchie (1998), Bradley et al. (2002), Comas e Moscardo (2005), Kang et al. (2005), Baloglu e Love (2005), Crouch e Louviere (2007) e Park et al. (2014). Esse critério foi considerado decisivo por Oppermann (1996a) em suas pesquisas, em contraposição ao constatado por Baloglu e Love (2005) quando os autores indicam que não houve considerações relevantes entre a experiência anterior e imagem do destino.

Visualiza-se que ASAE (1992) foi o estudo pioneiro ao inserir a imagem da localidade como atributo decisivo na escolha do destino, já Crouch e Ritchie (1998) foram os primeiros a trabalhar com os conceitos de reputação. Nota-se que após 1998 a maioria dos estudos inclui os atributos imagem e/ou reputação nas pesquisas, sendo que Edelstein e Benini (1994), Oppermann (1996a, 1996b), Qu et al. (2000), Bradley et al. (2002), Kim e Kim (2004), Kang et al. (2005), Chen (2006), DiPietro et al. (2008), Kim et al. (2011), Del Chiappa (2012), Huo (2014) e Park et al. (2014) trabalham com o conceito de imagem, enquanto Crouch e Ritchie (1998), Baloglu e Love (2003, 2005), Comas e Moscardo (2005), 
Crouch e Louviere (2007), Kim et al. (2010) apontam a reputação da cidade ao invés de indicar o atributo imagem. Apenas dois estudos fazem, diferenciação entre imagem e reputação trabalhando os dois atributos nas pesquisas (Huo, 2014; Kang et al., 2005).

Além do atributo imagem, ASAE (1992) inovou ao considerar o clima da localidade como atributo relacionado à escolha do destino, sendo que a maior parte dos outros estudos também aponta esse atributo (Baloglu \& Love, 2003, 2005; Chen, 2006; Comas \& Moscardo, 2005; Crouch \& Louviere, 2004, 2007; Crouch \& Ritchie, 1998; Del Chiappa, 2012; Edelstein \& Benini, 1994; Huo, 2014; Kang et al. 2005; Kim \& Kim, 2004; Kim et al. 2011; Oppermann, 1996a, 1996b; Park et al. 2014; Qu et al. 2000).

A acessibilidade também é um atributo citado na maior parte dos estudos Fortin, Ritchie e Arsenault (1976), ASAE (1992), Oppermann (1996a, 1996b), Crouch e Ritchie (1998), Qu et al. (2000), Bradley et al. (2002), Baloglu e Love (2003, 2005), Comas e Moscardo (2005), Kang et al. (2005), Chen (2006), Comas e Moscardo (2005), Crouch e Louviere (2007), Di Pietro et al. (2008), Del Chiappa (2012), Huo (2014), Park et al. (2014).

Oppermann (1996a) inovou nos atributos ao incluir os itens segurança e uma especificidade de entretenimento classificada como vida noturna. Quanto ao atributo segurança os estudos de Kim e Kim (2004), Kang et al. (2005), Crouch e Louviere (2007), Di Pietro et al. (2008), Kim et al. (2010), Del Chiappa (2012), Huo (2014) também o indicaram como decisivo. Os estudos de Kim e Kim (2004), Kim e Kim (2004), Kang et al. (2005) e Di Pietro et al. (2008) também destacaram o item vida noturna, assim como citado em Oppermann (1996a, 1996b).

O estudo pioneiro de Fortin, Ritchie e Arsenault (1976) já indicavam como um atributo decisivo ao se buscar um destino para eventos o apoio local, outros autores apontam esse mesmo atributo como pode ser visualizado em Crouch e Ritchie (1998), Kim e Kim (2004), Chen (2006), Di Pietro et al. (2008), Huo (2014). No mesmo sentido os estudos de Crouch e Ritchie (1998), Baloglu e Love (2003, 2005), Crouch e Louviere (2004), Comas e Moscardo (2005), Del Chiappa (2012), Huo (2014) apontam a importância das atividades e apoio do Convention Bureau ao se decidir sobre um destino. Apesar de o apoio local ser um atributo que pode trazer grande diferencial para a organização e um evento, seja pelas entidades relacionadas ao turismo ou a quem receberá o evento, nota-se que esse fator não é pesquisado na maioria dos estudos.

Após 1994, com o artigo de Edelstein e Benini (1994), outros 15 dos 17 conjuntos de atributos subsequentes apresentam claramente a preocupação com os custos. Confirmando o 
que Elston e Draper (2012) apontam, a preocupação com custos é predominante nos estudos, sendo que nos trabalhos de Oppermann (1996a, 1996b), Crouch e Ritchie (1998), Qu et al. (2000), Baloglu e Love (2003, 2005), Crouch e Louviere (2004, 2007), Kim e Kim (2004), Comas e Moscardo (2005); Kang et al.(2005), Chen (2006), Di Pietro et al. (2008), Kim et al.(2010), Kim et al. (2011), Del Chiappa (2012), Huo (2014), Park et al. (2014) encontra-se atributos nomeados como custo ou preço.

Questões de acesso ao destino do evento como visto, alfândega e demais barreiras que possam existir são apresentadas Crouch e Ritchie (1998) pela primeira vez, seguido por Kim e Kim (2004), Crouch e Louviere (2007) e Park et al. (2014). Esses fatores estão relacionados com a realização de eventos internacionais, podendo ser esse um dos motivos pelo qual o atributo não apresenta maior aderência aos conjuntos de atributos analisados. A mesma lógica pode ser aplicada ao atributo conhecimento em língua estrangeira, presente apenas nos artigos de Kim e Kim (2004), Huo (2014), Park et al. (2014).

Um atributo ainda pouco pesquisado, que apresentou crescimento no número de inclusões nos últimos anos, é a gentileza e hospitalidade da comunidade local. Ser bem recebido e quisto pelos moradores do destino em que o evento é realizado foi um atributo pesquisado por apenas Crouch e Ritchie (1998), Kim e Kim (2004), Comas e Moscardo (2005), Kang et al. (2005), Crouch e Louviere (2007), Kim et al. (2011) e Huo (2014).

Outro fator pouco pesquisado são os riscos para os eventos, fator esse que é citado por Crouch e Ritchie (1998), Comas e Moscardo (2005), Crouch e Louviere (2007). No mesmo sentido, a preocupação com a estabilidade política e social do destino é apontada como um fator que pode ser decisivo para o organizador ou promotor nos trabalhos de Kang et al. (2005) e Park et al. (2014).

Crouch e Ritchie (1998), Comas e Moscardo (2005) e Del Chiappa (2012) incluem um atributo que não é estudado em grande parte das pesquisas, mas que pode ser um diferencial na escolha de um local: a novidade do destino. Esse atributo pode ser importante para destinos que ainda estão fortalecendo o turismo de eventos e não são destinos eventuais. Considerar um destino novo pode ser um diferencial para a atratividade do evento.

Alguns atributos são apresentados de maneira pontual, como rentabilidade (Crouch \& Ritchie, 1998), promoção da associação (Crouch \& Ritchie, 1998), legislação local (Baloglu \& Love, 2003, 2005), atratividade para acompanhantes (Baloglu \& Love, 2003, 2005), tempo desde a última convenção no destino (Crouch \& Louviere, 2007), oportunidades de cooperação em pesquisa e desenvolvimento (Park et al., 2014). 
O conjunto de atributos mais sucinto dos estudos analisados é descrito por Kim et al. (2011) com 7 fatores associados. Em contraposição Crouch e Louviere (2007) apresentam 41 atributos, justificando que a escolha de um destino internacional envolve mais fatores do que quando o processo ocorre em território nacional.

Como apontado por Elston e Draper (2012), a sustentabilidade é um tema bastante relevante na atualidade que não está presente no conjunto de atributos dos estudos analisados. Ao se considerar a sustentabilidade de um destino ou espaço do evento responsável pelo evento pode ter além de ganhos ambientais a diminuição no uso de recursos e custos.

\section{CONSIDERAÇÕES FINAIS}

Com o objetivo de analisar os conjuntos de atributos considerados na escolha de um destino para evento pelos organizadores e promotores de eventos, esse artigo apresentou uma breve introdução sobre os temas turismo de eventos e tomada de decisão para basear a análise posterior dos artigos elencados na pesquisa bibliográfica.

Verificou-se que a tomada de decisão para escolha do destino por organizadores e promotores de eventos foi um tema trabalhado desde os anos 70 até a atualidade, porém sem regularidade das publicações e com um número reduzido de trabalhos. É importante ressaltar que no Brasil não foram encontrados trabalhos similares aos analisados, fato que aponta uma oportunidade para estudos e a necessidade de se trabalhar o tema.

De maneira geral, visualizou-se que os atributos podem ser categorizados entre aqueles que são relacionados ao evento ou ao destino. Nos modelos estudados, atributos como estrutura para eventos, hospedagem e custos estão presentes em grande parte dos conjuntos de fatores analisados, enquanto atributos como legislação, distância, facilidade de transporte, atratividade para o acompanhante, riscos para o evento, novidade do destino e a receptividade da comunidade local são atributos que aparecem isoladamente. Aponta-se a sustentabilidade como um fator que pode ser incluído como atributo em pesquisas futuras no conjunto de fatores que influenciam a tomada de decisão do organizador ou promotor de eventos sobre o destino.

É importante salientar que os atributos imagem e reputação do destino apresentaram grande incidência entre os estudos analisados, sendo que pelo menos um desses atributos aparece em 19 dos 21 conjuntos de atributos. A frequência com que o atributo é encontrado nos conjuntos estudados pode ser um indicativo de sua importância e, dessa forma, os atores 
envolvidos com o turismo de eventos devem estar atentos para desenvolver ações que possam trabalhar positivamente a imagem e reputação da localidade com o objetivo de a tornar mais atrativa.

A identificação dos atributos considerados pelos organizadores e promotores de eventos como essências na escolha de um destino e a avaliação desses atributos em estudos de caso são formas de apoio ao planejamento do turismo de eventos nos destinos receptores.

Esse estudo não teve o propósito de esgotar a discussão do tema, apenas incitar a avaliação crítica de um cenário através de um ensaio teórico. Sendo assim, sugere-se que outros estudos sejam elaborados sobre o tema para que se tenha um panorama do turismo de eventos no Brasil. Também é de suma importância que fatores como investimento no evento e número de participantes sejam correlacionados à importância de cada um dos atributos por esses fatores alterarem substancialmente a estrutura necessária à realização de um evento e às características que um destino deve apresentar.

\section{REFERÊNCIAS}

American Society of Association Executives (ASAE). (1992). Association meeting trends. Washington: ASAE.

Baloglu, S., \& Love, C. (2003). Association meeting planners' perceived performance of Las Vegas: an importance-performance analysis. Annals of the Journal of Convention \& Exhibition Management, 5(1), 13-27.

Baloglu, S., \& Love, C. (2005). Association meeting planners' perceptions and intentions for five major US convention cities: the structured and unstructured images. Tourism Management, 26(5), 743-752.

Bardin, L. (1977). Análise de conteúdo. Lisboa: Edições, 70.

Barretto, M. (2003). O imprescindível aporte das ciências sociais para o planejamento e a compreensão do turismo. Horizontes Antropológicos, 9(20), 15-29.

Bradley, A., Hall, T., \& Harrison, M. (2002). Selling cities: promoting new images for meetings tourism. Cities, 19(1), 61-70.

Bonn, M. A., Ohlin, J. B., \& Brand, R. R. (1994). Quality service issues: A multivariate analysis of association-meeting planner perceptions of Caribbean destinations. Journal of Hospitality \& Tourism Research, 18(1), 29-48.

Buhalis, D. (2000). Marketing the competitive destination of the future. Tourism management, 21(1), 97-116. 
Chacko, H. E., \& Fenich, G. G. (2000). Determining the importance of US convention destination attributes. Journal of Vacation Marketing, 6(3), 211-220.

Chen, C. F. (2006). Applying the analytical hierarchy process (AHP) approach to convention site selection. Journal of Travel Research, 45(2), 167-174.

Clark, J. D., \& McCleary, K. W. (1995). Influencing associations' site-selection process. Cornell Hospitality Quarterly, 36(2), 5-68.

Comas, M., \& Moscardo, G. (2005). Understanding associations and their conference decision-making processes. Journal of Convention \& Event Tourism, 7(3-4), 117-138.

Creswell, J. W. (2007). Projeto de pesquisa métodos qualitativo, quantitativo e misto. Ttrad. Luciana de Oliveira da Rocha (2a ed). Porto Alegre: Artmed.

Crouch, G. I., \& Louviere, J. J. (2004). The determinants of convention site selection: A logistic choice model from experimental data. Journal of travel research, 43(2), 118-130.

Crouch, G. I., \& Louviere, J. J. (2007). International Convention Site Selection: A further analysis of factor importance using best-worst scaling. Queensland: CRC for Sustainable Tourism.

Crouch, G. I., \& Ritchie, J. R. B. (1998). Convention site selection research: a review, conceptual model, and propositional framework. Annals of the Journal of Convention \& Exhibition Management, 1(1), 49-69.

Del Chiappa, G. (2012). How do meeting organizers choose convention sites based on different types of meetings? An empirical analysis of the Italian meetings industry. Event Management, 16(2), 157-170.

DiPietro, R. B., Breiter, D., Rompf, P., \& Godlewska, M. (2008). An exploratory study of differences among meeting and exhibition planners in their destination selection criteria. Annals of the Journal of Convention \& Event Tourism, 9(4), 258-276. Taylor \& Francis.

Domareski-Ruiz, T. C., Fernandes, D. L., \& Gândara, J. M. G. (2014). A contribuição do planejamento urbano na imagem e na competitividade do destino turístico de Curitiba (PR). Caderno Virtual de Turismo, 14(2), 150-166.

Edelstein, L. G., \& Benini, C. (1994). Meetings market report 1994. Annals of the Meetings \& Convention, 29(8), 60-82.

Elston, K., \& Draper, J. (2012). A review of meeting planner site selection criteria research. Annals of the Journal of Convention \& Event Tourism, 13(3), 203-220. Taylor \& Francis Group.

Filipovic, M. G. (2012). A new Prioritization method in the Analytic Hierarchy Process applied on a case study of the convention site selection. Annals of the Journal of Applied Mathematics, 3(2), 63-80. 
Fortin, P. A., Ritchie, J. B., \& Arsenault, J. (1976). A study of the decision process of North American associations concerning the choice of a convention site. Laval University: Quebec City.

Fratucci, A. C. (2014). Turismo e território: relações e complexidades. Anais do Caderno Virtual de Turismo, 14(1), 87-96.

Gândara, J. M. G., Ruiz, T. C. D., Chim-Miki, A. F., \& Biz, A. (2013). O Ciclo de Vida e o Posicionamento Competitivo dos Produtos Turísticos de Foz do Iguaçu desde a Perspectiva dos Atores Locais. Investigaciones Turísticas, (6), 1-26.

Getz, D. (2004). Bidding on events: Identifying event selection criteria and critical success factors. Annals of the Journal of Convention \& Exhibition Management, 5(2), 1-24. Taylor \& Francis Group.

Getz, D. (2008). Event tourism: Definition, evolution, and research. Tourism management, 29(3), 403-428.

Gil, A. C. (2007). Métodos e técnicas de pesquisa social (5a ed.). São Paulo: Atlas.

Huo, Y. (2014). Meeting Planners' Perception on Convention Destination Attributes: Empirical Evidence from Six Major Asian Convention Cities. The Journal of Business, 13(2), 70-80.

Jun, J., \& McCleary, K. W. (1999). Classifying US association meeting planners based on international destination selection criteria: A case study of South Korea. International Journal of Hospitality Management, 18(2), 183-199.

Kang, M. H., Suh, S. J., \& Jo, D. (2005). The competitiveness of international meeting destinations in Asia: Meeting planners' versus buying centers' perceptions. Annals of the Journal of Convention \& Event Tourism, 7(2), 57-85. Taylor \& Francis Group.

Kim, W. G., \& Kim, H. C. (2004). The analysis of Seoul as an international convention destination. Annals of the Journal of Convention \& Exhibition Management, 5(2), 69-87. Taylor \& Francis Group.

Kim, B. P., Kim, D., \& Weaver, P. A. (2010). An Exploratory Study of Local Organizations' Conference Choice Factors. Journal of Hospitality Marketing \& Management, 19(5), 503513.

Kim, S. S., Yoon, S., \& Kim, Y. (2011). Competitive positioning among international convention cities in the East Asian region. Annals of the Journal of Convention \& Event Tourism, 12(2), 86-105. Taylor \& Francis Group.

Mair, J., \& Thompson, K. (2009). The UK association conference attendance decision-making process. Tourism Management, 30(3), 400-409.

Miki, A. F. C., José, A. Z. C., Gândara, M. G., \& Muñoz, D. R. M. (2013). La variable competitividad en MeetingTourism: una visión general de la actualidad. Escenarios: empresa y territorio, 2(2), 131-154. 
Oppermann, M. (1996a). Convention destination images: analysis of association meeting planners' perceptions. Tourism management, 17(3), 175-182.

Oppermann, M. (1996b). Convention cities: Images and changing fortunes. Journal of tourism studies, 7(1), 10.

Oppermann, M., \& Chon, K. S. (1997). Convention participation decision-making process. Annals of the Tourism Research, 24(1), 178-191.

Park, J., Wu, B., Shen, Y., Morrison, A. M., \& Kong, Y. (2014). The Great Halls of China? Meeting Planners' Perceptions of Beijing as an International Convention Destination. Annals of the Journal of Convention \& Event Tourism, 15(4), 244-270. Routledge.

Qu, H., Li, L., \& Chu, G. K. T. (2000). The comparative analysis of Hong Kong as an international conference destination in Southeast Asia. Tourism Management, 21(6), 643-648.

Renaghan, L. M., \& Kay, M. Z. (1987). What meeting planners want: The conjoint-analysis approach. Cornell Hotel and Restaurant Administration Quarterly, 28(1), 66-76.

Van Raaij, W. F., \& Francken, D. A. (1984). Vacation decisions, activities, and satisfactions. Annals of the Tourism Research, 11(1), 101-112.

Wang, Y. (2008). Collaborative destination marketing: Roles and strategies of convention and visitors bureaus. Journal of Vacation Marketing, 14(3), 191-209. 